\title{
Uma Arquitetura Independente de Domínio e Plataforma para Apresentação de Comportamentos em Agentes Pedagógicos Animados
}

\author{
Ronaldo dos Santos Motola \\ Centro Universitários La Salle (Unilasalle) \\ ronaldomotola@yahoo.com.br \\ Patrícia Augustin Jaques \\ Programa Interdisciplinar de Pós-Graduação em Computação Aplicada (PIPCA) \\ pjaques@unisinos.br
}

\section{Resumo.}

Este artigo apresenta a arquitetura e implementação de um módulo corpo para agentes pedagógicos animados, ou seja, do módulo responsável por exibir os comportamentos verbais (falas) e corporais (animações) destes agentes. Esse módulo é independente de domínio e de aplicação, podendo ser inserido em outros ambientes educacionais voltados a outros domínios de aplicação, e independente de plataforma, possibilitando que possa ser executado em diferentes sistemas operacionais. Para tanto, ele foi implementado como um agente reativo desenvolvido em Java (o que possibilita que seja independente de plataforma), que se comunica com a mente utilizando a linguagem de comunicação entre agentes FIPA-ACL. Essa última capacidade do agente, permite que ele possa ser inserido em outros ambientes inteligentes de aprendizagem, desde que estes possuam a capacidade de se comunicar com ele via FIPA-ACL.

Palavras-chaves: agente, agente pedagógico animado, corpo do agente e FIPA

\section{An Independent Architecture of Domain and Platform for Presentation of Behaviors in Animated Pedagogical Agents.}

\begin{abstract}
.
This article presents the architecture and the implementation of the module body for animated pedagogical agents, that is, the responsible module for showing the verbal (speak) and corporal (animation) behaviors of these agents. This module is independent of domain and application, being able to be inserted in other educational environments dedicated to other application domains, and independent of platform, making possible that it can be executed in different operational systems. In this way, it was implemented as a reactive agent in Java (what makes it independent of platform), that communicated with the mind using the communication language among agents FIPA-ACL. This last capacity of the agent, allows it to be inserted in other intelligent learning environments, since they are able to communicate in FIPA-ACL
\end{abstract}

Keywords: agent, animated pedagogical agent, agent's body and FIPA 


\section{Introdução}

Os Sistemas Educacionais à Distância Mediados por Computador (EDMC) vêm crescendo nos últimos anos, assim como a utilização da Inteligência Artificial em suas aplicações. Segundo Giraffa (1999) tem-se observado uma crescente investigação por parte dos pesquisadores na área de Inteligência Artificial devido às potencialidades das tecnologias disponibilizadas. Uma dessas tecnologias, os agentes inteligentes, abre novas possibilidades no que diz respeito à interação aluno e tutor.

Por outro lado, nota-se que o índice de evasão ainda é muito elevado devido, em grande parte, à falta de incentivo e motivação ao aluno. Segundo BORBA (2006), as instituições de Educação à Distância (EAD) têm se preocupado mais com a questão tecnológica e deixando de lado a questão afetiva com os alunos. Para ele, os alunos acabam desistindo dos cursos por causa da falta de laços afetivos entre eles, os colegas e professores. Os agentes pedagógicos animados podem ser um importante recurso nesses ambientes, motivando e auxiliando o aluno em suas atividades.

Os Agentes Pedagógicos Animados são agentes que tem um papel educacional ou pedagógico para facilitar ou aperfeiçoar a aprendizagem do aluno e que são representados por um personagem animado que interage com o aluno. Estes agentes usam recursos de multimídia para fornecer ao usuário um personagem animado com características semelhantes àquelas de seres vivos inteligentes. (JAQUES, 2000). Além disso, esses agentes devem possuir uma interface amigável estimulando o aluno a aprender e evitando a evasão nessas atividades.

Para Johnson (1999) os Agentes Pedagógicos Animados (APA) possuem duas vantagens nos ambientes educacionais: aumentam a capacidade de comunicação entre os estudantes e o computador, assim como, atraem a atenção dos alunos e os motivam.

Segundo ele, os APAs são um novo paradigma para os ambientes de ensino, por estarem aptos a demonstrarem tarefas complexas, além de empregarem locomoção e possuírem gestos para focar a atenção dos alunos.

A arquitetura dos agentes pedagógicos animados é geralmente composta por dois módulos: a Mente (responsável pela modelagem do aluno e escolha das táticas) e o Corpo, responsável por exibir os comportamentos verbais (falas) e corporais (animações) destes agentes. Embora a mente do agente deva ser concebida especificamente para o ambiente onde o agente será inserido, considerando o perfil do aluno, o domínio do sistema e as teorias pedagógicas que embasam esse sistema, entre outros, as funcionalidades do módulo corpo são, de forma geral, as mesmas em todos os agentes. Esse módulo deve receber uma tática da mente e aplicá-la escolhendo os comportamentos verbais e corporais adequados. Porém, geralmente, é necessário desenvolver uma nova implementação ou modelagem deste módulo, visto que na grande maioria dos casos, são utilizadas tecnologias dependentes de plataforma, ou estes são concebidos para uma determinada aplicação e dependentes desta.

O propósito deste trabalho é desenvolver um módulo Corpo que seja independente de domínio e plataforma, de maneira que possa ser utilizado também em outros ambientes. Dessa forma, a mente do agente também poderá ser desenvolvida em outras plataformas e ou linguagens de implementação. Para que esse módulo seja independente de domínio e de aplicação, podendo ser inserido em outros ambientes educacionais voltados a outros domínios de aplicação, e independente de plataforma, possibilitando que possa ser executado em diferentes sistemas operacionais, esse módulo foi implementado como um agente reativo desenvolvido em Java (o que possibilita que seja independente de plataforma), que se comunica com a mente utilizando a linguagem de comunicação entre agentes FIPA-ACL. Essa última capacidade do agente permite que ele possa ser inserido em 
outros ambientes inteligentes de aprendizagem, desde que estes possuam a capacidade de se comunicar com ele via FIPA-ACL.

\section{Metodologia}

Esse trabalho foi realizado em três fases principais. Na primeira fase foi realizada uma pesquisa sobre as tecnologias existentes para implementação do personagem animado.

Uma vez que o projeto está sendo desenvolvido na Linguagem Java, o que lhe permite ser independente de plataforma, primeiramente foi estudado Java Speech API e Java 3D API que tratam do Reconhecimento de Voz, do Sintetizador de Voz e de desenvolvimento de objetos 3D. Além dessas ferramentas para implementação, foram estudadas também o Framework FIPA-OS, que servirá para a comunicação com outros agentes.

A fase seguinte tratou da definição da aparência do personagem animado. Para isso foi preciso realizar algumas entrevistas com profissionais pedagogos para elaboração de um questionário adequado, para ser aplicado em professores e alunos de Escolas de Ensino Fundamental de $3^{\mathrm{a}}$ e $4^{\mathrm{a}}$ série. Conforme os resultados desse questionário, foi definida a aparência desse personagem. Após essa fase, procurou-se um profissional na área de designer para desenhar os movimentos em um arquivo gráfico que compõem as animações dos comportamentos do personagem.

$\mathrm{Na}$ fase final, foi realizada a definição da arquitetura do módulo corpo como um agente reativo, bem como a modelagem deste módulo em UML (Unified Modeling Language) e a implementação utilizando a Linguagem JAVAтм. Como a comunicação entre agentes será realizada seguindo os padrões FIPA, utilizou-se o Framework FIPAOS.

Para testar esse módulo, foi desenvolvido um ambiente simples sem a parte inteligente (mente do personagem), para demonstrar as funcionalidades do Agente Animado, uma vez que, não é objetivo deste trabalho implementar a Mente do Personagem.

\section{Definição da aparência do agente animado}

Inicialmente, para a definição da aparência do personagem animado, foi preciso realizar algumas entrevistas com pedagogos para elaboração de um questionário adequado, para ser aplicado em professores e alunos de Escolas de Ensino Fundamental de $3^{\mathrm{a}}$ e $4^{\mathrm{a}}$ série. O objetivo desse questionário era definir a aparência do personagem que fosse adequada a esse perfil de alunos. Conforme os resultados obtidos através desse questionário, podem-se tirar as seguintes conclusões:

- Quanto ao tipo de personagem: deve possuir mais de um personagem, pelo fato das respostas serem muito variadas, deixando para o usuário escolher o tipo de personagem;

- Quanto à aparência: deve possuir uma aparência de intelectual e alegre;

- Quanto ao tamanho: deve possuir aproximadamente $10 \mathrm{~cm}$ e de corpo inteiro;

- Quanto ao comportamento: deve possuir comportamentos variados, como por exemplo, incentivo, ajuda e saudação entre outros, assim o personagem terá mais de uma animação para cada tipo de comportamento;

- Quando a interação com o aluno: através de gestos e fala, deixando o aluno optar entre ocultar e exibir o personagem e também deixar o personagem mudo ou não.

Com base no questionário observou-se que as respostas eram muito variadas no que diz respeito à aparência do personagem, assim optou-se por ter mais de um personagem 
deixando a critério do usuário escolher. Essa opção foi inclusive apontada pelos próprios pedagogos e professores entrevistados neste trabalho e nos trabalhos de Bocca (2003) e Jaques (2005). Para isso foi desenvolvido um ambiente onde o tutor poderá cadastrar diversos personagens juntamente com seus comportamentos.

Nas Figura 1, Figura 2 e Figura 3, apresentamos três personagens iniciais que foram utilizados para elaboração do trabalho. Porém, o sistema permitirá que sejam adicionados outros personagens, assim como os comportamentos físicos e verbais de cada um.

Os personagens e os seus comportamentos foram desenvolvidos pelo designer Jonatan Sarmento, colaborador voluntário do projeto.

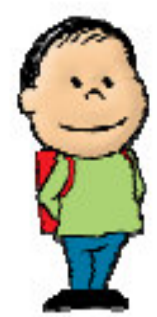

Figura 1 - Personagem: Jeff

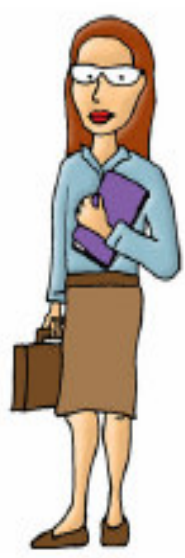

Figura 2 - Personagem: Maria

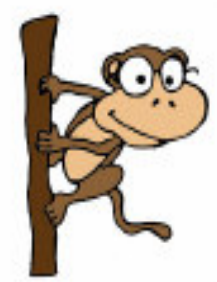

Figura 3 - Personagem: Monkey

\section{Modelo Proposto}

Visualizamos os módulos corpo e mente de uma arquitetura de agente pedagógico animado como agentes autônomos e independentes que se comunicam. A Mente é um agente inteligente cognitivo, que realizará todo o trabalho de modelagem cognitiva e afetiva do aluno, bem como, baseado nesse modelo de aluno irá determinar as estratégias e táticas a serem aplicadas. Por outro lado, o Corpo será um agente reativo, que conforme a tática 
escolhida pela Mente apresentará um comportamento físico e verbal que corresponda à tática escolhida. Com isso uma questão importante é a comunicação desse módulo com a mente, a qual será discutida na seção 4.2. Na próxima seção (4.1) será apresentada a arquitetura do módulo corpo.

\subsection{Arquitetura do Módulo Corpo}

O módulo mente deverá gerar uma tática a ser adotada e enviar esta como mensagem para o Corpo. Este por sua vez, ao receber a mensagem, faz um tratamento para capturar a tática da mensagem e, então, a envia ao componente Gerenciador de Comportamentos (GC). O GC escolhe na Base de Dados (BD) os comportamentos que fazem parte da tática e envia ao componente Animador. Essa seleção não é feita de forma aleatória.

Para isso, uma vez selecionado o comportamento, é realizado uma verificação na data de sua última utilização, cuja informação consta na $\mathrm{BD}$, evitando a repetição de comportamentos.

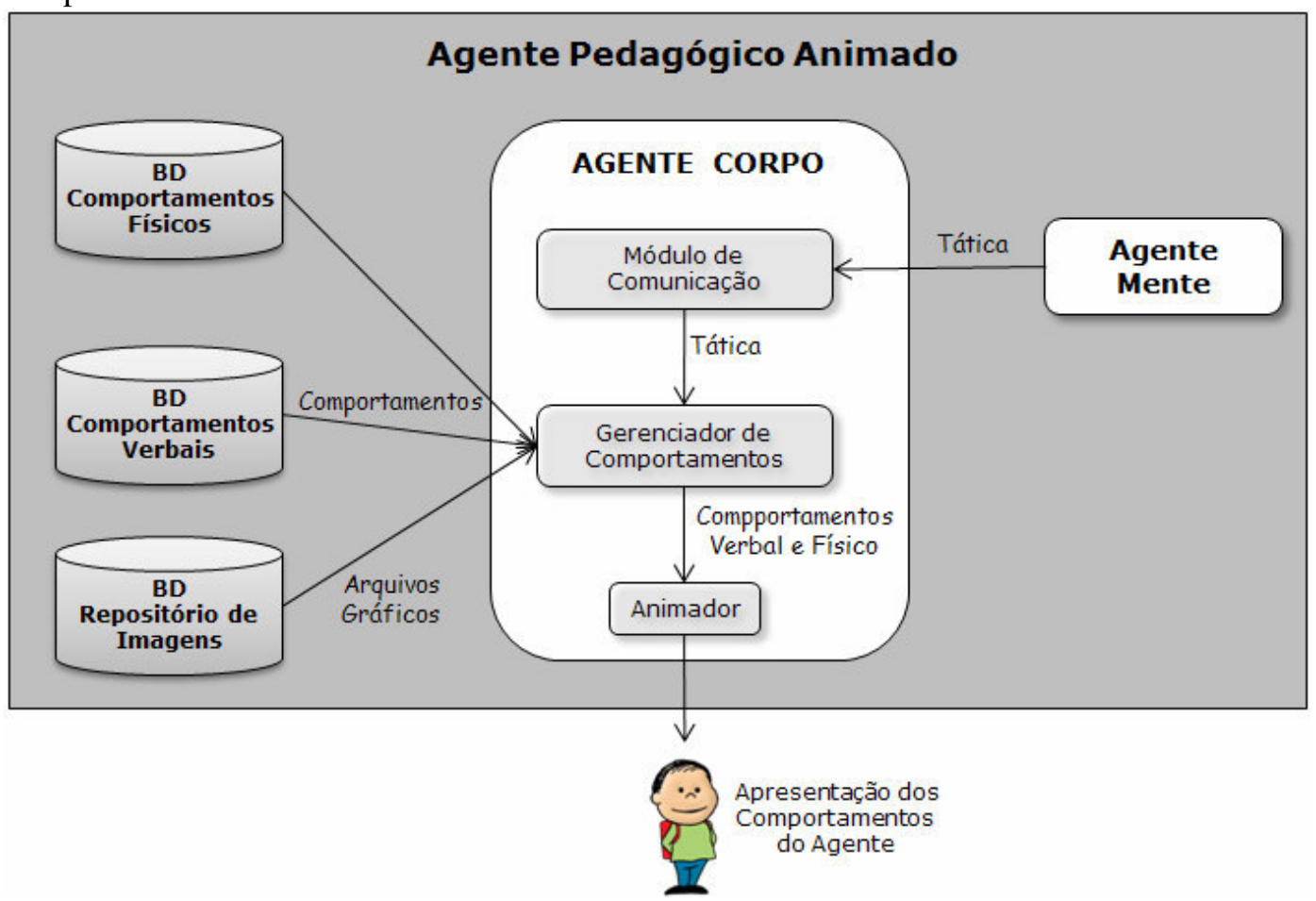

Figura 4 - Arquitetura Interna do Corpo do Agente

À geração da animação é realizada pelo componente Animador. Esse componente é formado por classes desenvolvidas por WILGES (2006) utilizando a Linguagem Java e será descrito nas seções que seguem. O Animador é responsável pela manipulação dos arquivos gráficos contendo as animações que fazem parte de um comportamento e também pela apresentação da animação na tela do usuário. Os arquivos gráficos estarão em um repositório chamado Imagens. Conforme pode ser observado na Figura 5, a pasta PROFESSORA, por exemplo, representa o personagem, as subpastas Aplauso, Caminhar e Telefonar representam os comportamentos deste personagem, e os arquivos aplauso00 à aplauso10 são os arquivos gráficos que serão usados para gerar dinamicamente o comportamento aplauso. Essa arquitetura do agente reativo corpo pode ser observada na Figura 4.

Sendo assim, todos os Personagens, juntamente com seus respectivos comportamentos, estarão instalados na pasta imagens do diretório corrente do sistema. 


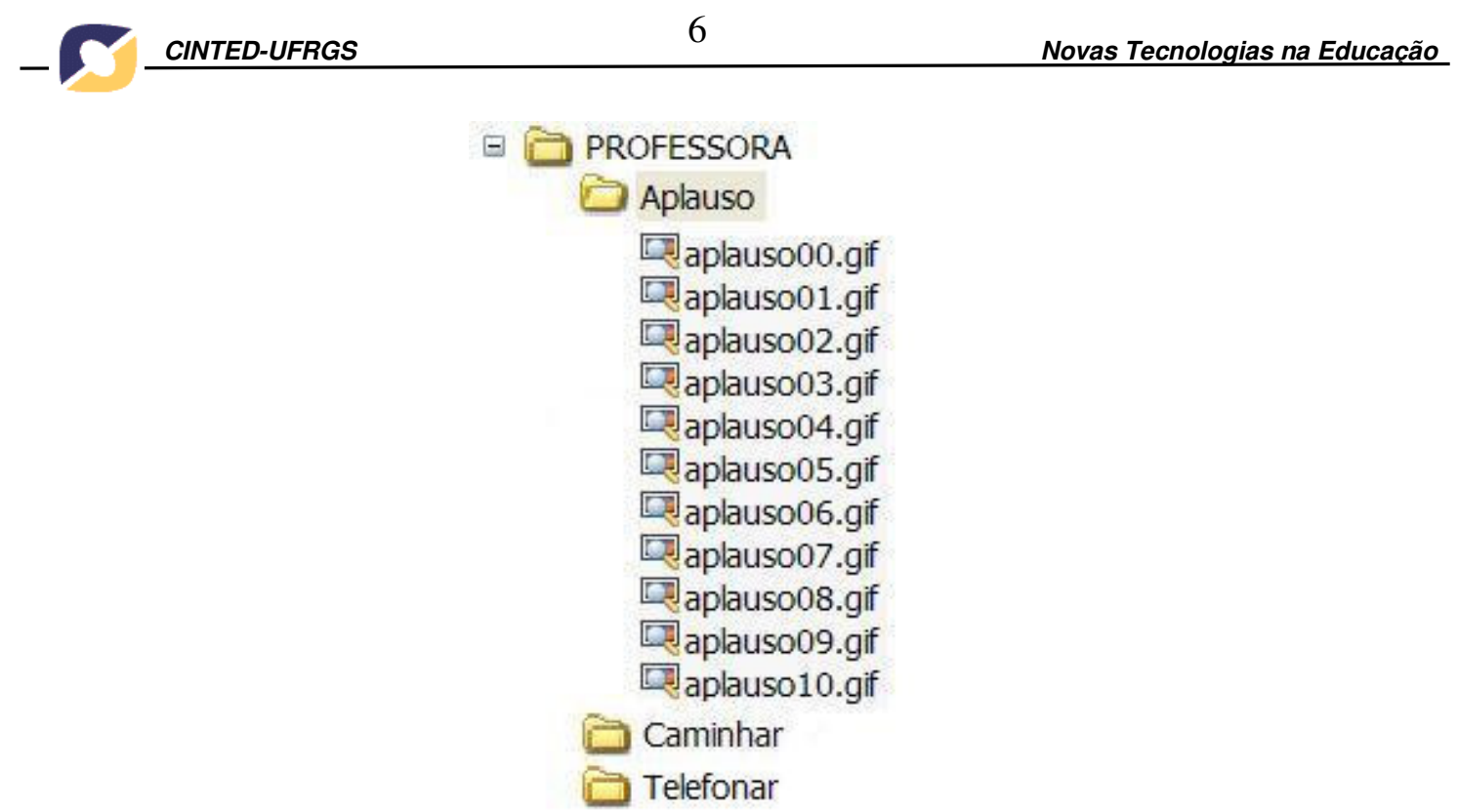

Figura 5 - Estrutura do repositório Imagens

\subsection{Comunicação com outros agentes}

A comunicação com outros agentes é realizada seguindo os padrões FIPA. Para isso foi utilizado o Framework FIPA-OS 1 por ser desenvolvido utilizando a linguagem Java e possuir todos os recursos de comunicação que a FIPA estabelece.

Para que a comunicação do agente com os demais seja realizada, é preciso configurar alguns parâmetros para a Linguagem de comunicação (ACL). Um exemplo de mensagem recebida pelo agente Corpo é:

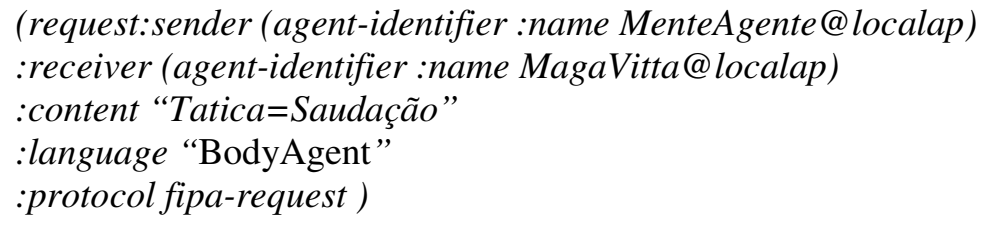

A mensagem acima representa uma mensagem recebida da mente, cujo AID é MenteAgente@localap. Essa mensagem possui como conteúdo (content) a tática de "Saudação", a linguagem de comunicação (language) é "BodyAgent" e seu protocolo de iteração (protocol) é o "fipa-request". O agente corpo ao receber essa mensagem, escolhe um comportamento baseado no conteúdo da mensagem.

\subsection{Componentes do sistema proposto}

O Sistema possui alguns componentes importantes para sua execução. São eles:

- O Framework FIPA-OS: esse deverá ser instalado e configurado em cada estação de trabalho. Ele é responsável pela comunicação do agente corpo com o agente mente.

- A Base de Dados: contendo as informações do agente.

- O Agente Reativo Corpo: São as classes do Agente Corpo que deverão ser configuradas na plataforma FIPA-OS.

- O Repositório das Animações: São os arquivos gráficos contendo os comportamentos Corporais de cada Personagem. Esse repositório é representado pela pasta Imagens do diretório corrente do Agente.

1 Disponível para download em: http://sourceforge.net/projects/fipa-os/ 
Esses componentes fazem parte do pacote do sistema e deverão ser instalados em cada estação.

\subsection{Aspectos de Implementação}

O sistema todo foi organizado em três pacotes de acordo com suas funcionalidades:

Gerenciador, Comportamento e Base. O pacote Gerenciador possui as classes principais do sistema, ou seja, classes que fazem o gerenciamento e manipulação dos objetos do sistema. Já no pacote Comportamento, estão agrupados os objetos do sistema, como por exemplo, os objetos Personagem, Tática, Comportamento Verbal e Comportamento Corporal. O pacote Base possui as classes que manipulam os dados diretamente da Base de Dados.

Além disso, o sistema como um todo, necessita ainda de uma Base de Dados, onde armazena informações importantes para a execução do sistema. Assim, o Tutor responsável pelo ambiente educacional em que esse agente irá atuar, possui o papel de executar as atualizações nessa BD. A BD é composta por seis tabelas: Personagem, Tatica, CCorporal, CVerbal, AcaoCorporal e AcaoVerbal.

Conforme pode ser observado na Figura 6, os Comportamentos Corporais estão relacionados com a tabela Tática através da tabela AcaoCorporal, assim como os

Comportamentos Verbais estão relacionadas com a tabela Tática através da tabela AcaoVerbal. Para cada Tática pode existir mais de um comportamento físico e verbal. Sendo assim, o sistema faz uma busca do comportamento mais antigo através da data de sua última utilização. Outra informação importante na tabela CCorporal é o endereço do repositório a qual foi instalado os comportamentos, para que ao gerar a animação, o sistema possa buscar os arquivos gráficos necessários desse comportamento.

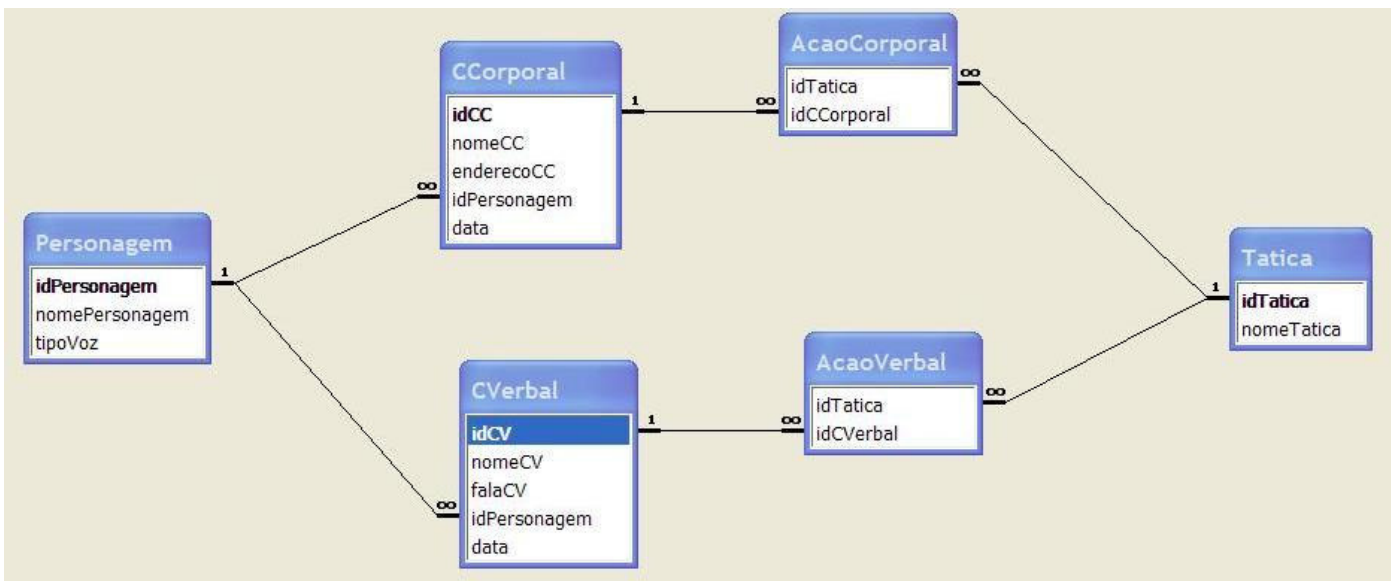

Figura 6 - Relacionamentos das Tabelas

\section{Considerações Finais}

Os testes desse projeto foram realizados através de um ambiente simples sem a parte inteligente (mente do personagem), para demonstrar as funcionalidades do Agente Animado, uma vez que, a Mente do Personagem será implementada em trabalhos futuros com alguma tese de Mestrado ou Doutorado, fazendo então a validação propriamente dita de todo o Agente Pedagógico. O ambiente de teste foi desenvolvido utilizando as técnicas de comunicação entre Agentes.

Como melhorias futuras para o projeto pensa-se em utilizar arquivos do tipo XML para a persistência dos objetos do sistema no lugar de uma Base de Dados, deixando assim o sistema mais otimizado e independente de plataforma. A utilização de arquivos XML 
possibilitaria o uso do agente em micro ambientes como, por exemplo, em celulares para deficientes auditivos.

Outra melhoria que pode ser considerada é a criação de um atualizador dos comportamentos. Esse atualizador poderia ser no próprio ambiente do tutor onde, após a inclusão de um novo comportamento no servidor, seria realizada automaticamente a atualização nas estações de trabalho cujo agente esteja atuando.

Além disso, outras idéias de trabalhos futuros são previstas:

- Reconhecimento de voz natural, propiciando assim um maior realismo ao agente no que diz respeito à interação com o aluno;

- A utilização dos movimentos corporais dos agentes pedagógicos animados para estes se comunicarem em LIBRAS (Linguagem Brasileira de Sinais) através de suas expressões corporais, com alunos com necessidades especiais.

\section{Referências Bibliográficas}

BOCCA, Everton. Modelagem e Implementação da Interface para Apresentação de Comportamentos Animados e Emotivos de um Agente Pedagógico Animado.

2003. Dissertação (Mestrado em Ciência da Computação) - Instituto de Informática, UFRGS, Porto Alegre.

BORBA, Jean Marlos P.(2006). Como desenvolver afetividade com o aluno de

EAD.

Disponível em: http://www.universia.com.br/materia/materia.jsp?materia=10566 Acessado em: 01 ago. 2006.

FIPA-OS. FIPA-OS Developers Guide. Disponível em: http://fipaos. sourceforge.net/tutorials.htm. Acessado em: 01 set. 2006.

GIRAFFA, Lúcia M. M. Uma arquitetura de tutor utilizando estados mentais. 1999.

Tese (Doutorado em Ciência da Computação) - Instituto de Informática, UFRGS, Porto Alegre.

JAQUES, Patrícia et al. Interação com Agentes Pedagógicos Animados: Um Estudo Comparativo. IHC 2001 - IV Workshop on Human Factors in Computer Systems. 15 a 17 de outubro de 2001, Florianópolis, SC.

JAQUES, Patricia; VICCARI, Rosa. PAT: Um agente pedagógico animado para interagir afetivamente com o aluno. Revista Novas Tecnologias na Educação Maio/2005, vol. 3, no 1. Disponível em: http://www.cinted.ufrgs.br/renote/maio2005/ Acessado em: 20 abr. 2006.

JOHNSON, L. et al. Pedagogical Agentes on The Web. In: ITS'98 Workshop on Pedagogical agents, 4,1998. San Antônio. Anais. San Antonio:1998.p.2-7.

Disponível em http://www.isi.edu/isd/ADE/papers/its98/ITS98-WW.htm Acessado em: 20 abr. 2006.

WILGES, Beatriz. Um Agente Pedagógico Animado no papel de LMS Manipulando Objetos Inteligentes de Aprendizagem. 2006. 71 f. Trabalho de Conclusão - Curso de Bacharelado em Ciência da Computação, Departamento de Informática, UFPEL, Pelotas, 2006. 